\title{
Survival of Escherichia coli in the environment: fundamental and public health aspects
}

Jan Dirk van Elsas, Alexander V Semenov, Rodrigo Costa and Jack T Trevors

The ISME Journal (2011) 5, 367; doi:10.1038/ismej.2010.187

Correction to: The ISME Journal (2011) 5, 173-183; doi:10.1038/ismej.2010.80; published online 24 June 2010

The authors of the above article have noticed a misprint in the publication of their paper. This occurs in the second sentence of the introduction. The sentence should read: 'On the basis of genomic information, the species has been divided into five (four major) different phylogenetic groups, denoted as A, B1, B2, D and E (Touchon et al., 2009).'

The authors would like to apologize for any inconvenience this may have caused. 